\title{
Recurrent Transient Loss of Consciousness Induced by Acute Massive Gastrointestinal Hemorrhage during Hemodialysis: A Case Report
}

\author{
Han Li Shi-xiang Wang \\ Blood Purification Center, Beijing Chao-Yang Hospital, Capital Medical University, Beijing, China
}

\section{Key Words}

Transient loss of consciousness $\cdot$ End-stage renal disease $\cdot$ Massive gastrointestinal hemorrhage

\begin{abstract}
A 67-year-old man with chronic hemodialysis (HD), who had end-stage renal disease secondary to diabetic nephropathy and had been on HD for 9 years with ultrapure dialysis solution, is presented with recurrent transient loss of consciousness during HD.

Copyright $\odot 2010$ S. Karger AG, Base
\end{abstract}

\section{Case Report}

On May 8, 2010, in the morning, a 67-year-old man on chronic hemodialysis (HD) with predialysis blood pressure 145/85 mm Hg and heart rate 62 beats/min presented with convulsion and unconsciousness $30 \mathrm{~min}$ after starting HD. He had end-stage renal disease (ESRD) secondary to diabetic nephropathy and had been on HD for 9 years with an ultrapure dialysis solution schedule of 3 sessions per week. He had no hemorrhagic tendency and no peptic ulcer. A routine heparin, constant-infusion method was used during HD. He was given an initial 15$\mathrm{mg}$ heparin bolus dose followed by $7.5 \mathrm{mg} / \mathrm{h}$ infusion for $3 \mathrm{~h}$. He had had diabetes mellitus for 25 years and hypertension for 9 years. His usual predialysis blood pressure was 140-160/80-90 mm Hg. On May 6, 2010, he had un- dergone a conventional blood analysis, which is done once monthly in chronic HD patients. Laboratory values showed: Hb 106 g/l, Hct 32.7\%, WBC 7.52 $10^{9} / 1$, PLTs $297 \cdot 10^{9} / 1$, serum creatinine $1,037.1 \mu \mathrm{mol} / \mathrm{l}$, serum BUN $23.65 \mathrm{mmol} / \mathrm{l}$, and serum potassium $4.9 \mathrm{mmol} / \mathrm{l}$. An initial physical examination revealed that he suffered from obnubilation. However, pathological signs of the nervous system were negative and both of his pupils were isocoric. His vital signs were: blood pressure $92 / 50 \mathrm{~mm} \mathrm{Hg}$, respiratory rate 20 breaths $/ \mathrm{min}$, and heart rate 100 beats $/ \mathrm{min}$. His blood glucose concentration was $5.8 \mathrm{mmol} / \mathrm{l}$ by glucometer. The ultrafiltration rate was immediately reduced to zero and a bolus of $0.9 \%$ saline $(100 \mathrm{ml})$ was rapidly administered. After the above-mentioned treatment, his blood pressure returned to $105 / 60 \mathrm{~mm} \mathrm{Hg}$ and consciousness returned to normal. However, he then presented with another conscious disturbance after HD ultrafiltration was resumed. HD was stopped and he was transferred to our emergency department. His consciousness returned to normal again after HD ultrafiltration was stopped and a bolus of $0.9 \%$ saline $(500 \mathrm{ml})$. A computed tomographic (CT) scan of his head revealed normal findings. An electrocardiogram and head ultrasound also revealed normal findings. His serum $\mathrm{LDH}$, haptoglobins, and red cell fragments were all normal, but serum potassium and BUN were 7.8 and $36.07 \mathrm{mmol} / \mathrm{l}$, respectively which were higher than 2 days earlier. He was therefore treated with urgent heparin-free HD again (in order to avoid intracerebral hemorrhage) on that same day in the afternoon. He presented with convulsion and

\section{KARGER}

Fax +4161306 1234 E-Mail karger@karger.ch www.karger.com
(C) 2010 S. Karger AG, Basel

0253-5068/10/0303-0178\$26.00/0

Accessible online at:

www.karger.com/bpu
Shi-xiang Wang

Blood Purification Center, Beijing Chao-Yang Hospital

Capital Medical University, No. 8 Gongti South Road

Beijing 100020 (China)

Tel./Fax +86 106593 5007, E-Mail wxy1988@263.net 
1

Fig. 1. Gastroscopic morphological change of multiple superficial ulcers in the antrum.

Fig. 2. Gastroscopic morphological change of mucous hyperemia and mucosal edema around the ulcers in the antrum.
2

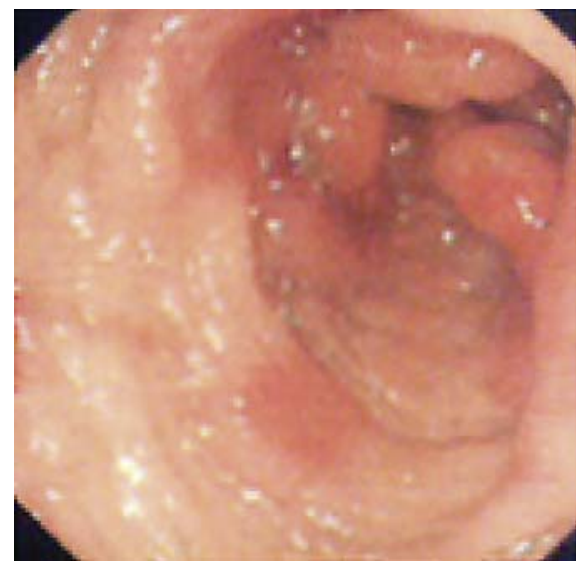

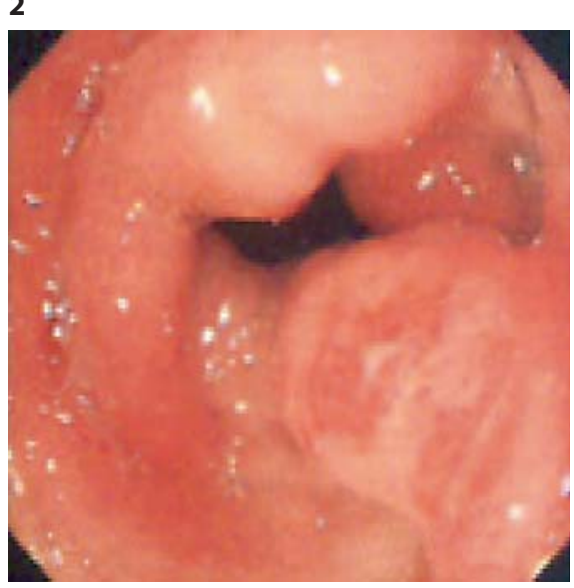

unconsciousness again 60 min after HD was initiated. HD was stopped and he was transferred to our emergency department again, where his consciousness returned to normal after a massive fluid infusion.

His blood cell count was not remeasured until the morning of May 10, 2010, when he was observed to have anemia during the next session of HD. During the same morning, his blood cell count revealed severe anemia: RBC $1.07 \cdot 10^{12} / 1$, Hb 33 g/l, Hct 10.7\%, WBC $24.61 \cdot 10^{9} / 1$, and PLTs $220 \cdot 10^{9} / 1$. After careful questioning, he complained of large unshaped dark stools on the morning of May 8, 2010, without abdominal pain, and after that, did not have any stools until May 16, 2010. His abdomen was flat and soft, with no tenderness. His liver and spleen were not palpable. On May 16, 2010, the routine stool test showed that occult blood was positive and there was RBC in his stools. It is worth noting that his stools were brownish in color and the occult blood test was positive after beginning treatment with ferrous succinate $200 \mathrm{mg} 3$ times a day for anemia for 9 years. He also had a history of positive HCV antibody for 9 years, but the level of HCV-RNA was negative. Serum liver biochemistry, amylase and lipase were normal. Ultrasound of the upper abdomen revealed a normal gallbladder, liver and spleen, but a thickened gastric wall, suggestive of gastritis. Upper gastrointestinal endoscopy showed multiple superficial ulcers in the antrum of the stomach with a 5- to $6-\mathrm{mm}$ diameter along with mucous hyperemia and mucosal edema around the ulcers (fig. 1,2). Multiple antral biopsies were taken and there was no evidence of malignancy. A Camplyobacter-like organism test for Helicobacter pylori yielded a negative result.

He had no previous personal or family history of ulcer disease and was not on aspirin or non-steroidal anti-in- flammatory drugs. Uremia-induced gastric ulceration seemed to be the most probable diagnosis. He was treated with RBC transfusion and oral omeprazole $20 \mathrm{mg}$ daily and heparin-free HD. On May 23, 2010, his routine stool test showed that occult blood was negative and there was no RBC in his stools.

\section{Discussion}

A 67-year-old man with chronic HD who presented with recurrent transient loss of consciousness during HD is reported. The most common causes of acute cerebral dysfunction during or immediately after $\mathrm{HD}$ are disequilibrium syndrome and intracranial bleeding $[1,2]$. Because the possibility of disequilibrium syndrome is seldom seen in patients on regular HD for 9 years, the cause of his recurrent transient loss of consciousness during HD was simply and incorrectly diagnosed as an acute cerebrovascular accident. It was not until the result of his head CT scan revealed normal findings that the possibility of an acute cerebrovascular accident was excluded. An ultrapure dialysis solution was used and the hemodialyzers were not reused in our unit. Only this patient presented a drop in Hct on that particular day, and his serum $\mathrm{LDH}$, haptoglobins, and RBC fragments, which were examined in the emergency room, were all normal. Also, the rapidity of hyperkalemia was caused by decomposing and reabsorption of blood in the gastrointestinal tract, so it was not a possible explanation that his drop in Hct was caused by hemolysis. The real cause of his recurrent transient loss of consciousness during HD was hypovolemia due to an acute massive gastrointestinal hemorrhage. The attending emergency room doctor was misled by the patient's nor- 
mal blood cell count 2 days earlier. The diagnosis of severe anemia due to massive blood loss was missed; however, in the early stage of acute massive blood loss, the level of hemoglobin, RBC count and erythrocrit were usually normal. A prompt and correct diagnosis of acute massive gastrointestinal hemorrhage after a careful physical examination and blood test are of utmost importance.

Gastrointestinal bleeding is occurring with a greater frequency and is associated with a higher mortality in uremic than non-uremic patients [3, 4]. In the US Renal Data System study of renal transplant recipients, the ageadjusted risk ratio (RR) for hospitalization due to gastrointestinal bleeding is 10.7 compared with that in the general population. Upper gastrointestinal bleeding accounts for 3-7\% of all deaths in patients with ESRD [5]. A prospective study by Fiaccadori et al. [6] found that acute gastrointestinal hemorrhage in patients with impaired renal function was associated with an increase in mortality $(\mathrm{RR}=2.57)$ and a $37 \%$ increase in length of hospital stay as compared to non-renal failure patients. In patients with ESRD, the most common causes of upper gastrointestinal bleeding are peptic ulcers, hemorrhagic esophagitis, gastritis, duodenitis and gastric telangiectasias $[7,8]$. This patient did not have any gastrointestinal symptoms, except gastric ulceration. Endoscopy confirmed the presence of a significant mucosal injury. There was no personal or family history of ulcer disease. There were, however, some clues with respect to the gastrointestinal hemorrhage in this patient, such as high blood urea nitrogen and hyperpotassemia even after dialysis because of toxin resorption after gastrointestinal hemorrhage.
On the other hand, renal anemia is very common in maintenance HD patients as a result of the insufficient synthesis of EPO [9]. However, use of rHuEPO increases iron demands. It is demonstrated that in more than half of the patients on rHuEPO therapy, erythropoiesis will be affected by iron deficiency [10]. Although iron storage may be normal or even increased in some patients, many lack available iron since the iron stored cannot be effectively released to meet the demands of the bone marrow for hemopoiesis. This may cause functional iron deficiency and may also reduce the efficacy of anemia therapies [11]. According to guidelines [12], all anemic, iron-deficient HD patients should be administered iron. After oral iron, all patients have dark stools and routine stool tests show that occult blood is positive without gastrointestinal bleeding because of the effect of oral iron. The use of intravenous iron is therefore recommended in maintenance HD patients with an iron deficiency to avoid the diagnosis of gastrointestinal hemorrhage.

\section{Acknowledgement}

We thank nurse Wan-ci Zhang in the Blood Purification Center of Beijing Chao-Yang Hospital, Capital Medical University, for her help with the collection of clinical data.

\section{Disclosure Statement}

The authors have no potential conflicts of interest to be disclosed. This article was not supported by any financial grants.

\section{References}

1 Patel N, Dalal P, Panesar M: Dialysis disequilibrium syndrome: a narrative review. Semin Dial 2008;21:493-498.

$\checkmark 2$ Watanabe A: Cerebral microbleeds and intracerebral hemorrhages in patients on maintenance hemodialysis. J Stroke Cerebrovasc Dis 2007;16:30-33.

3 Jaminidar PKB: Gastrointestinal disease in patients with chronic renal failure; in Nissenson AR (ed): Clinical Dialysis. East Norwalk, Appleton \& Lange, 1995, vol 3, chapt 26, pp 607-617.

4 Toke AB: Gastrointestinal bleeding risk in patients undergoing dialysis. Gastrointest Endosc 2010;71:50-52.
5 Boyle JM, Johnston B: Acute upper gastrointestinal hemorrhage in patients with chronic renal disease. Am J Med 1983;75:409-412.

6 Fiaccadori E, Maggiore U, Clima B, Melfa L, Rotelli C, Borghetti A: Incidence, risk factors, and prognosis of gastrointestinal hemorrhage complicating acute renal failure. Kidney Int 2001;59:1510-1519.

7 Furkert JD, Zeier M, Schwenger V: Gastrointestinal hemorrhage in hemodialysis patients. Z Gastroenterol 2008;46:1266-1269.

$\checkmark 8$ Chandra A, Tso R, Cynamon J, Miller G: Massive upper gastrointestinal bleeding in a long-term hemodialysis patient. Chest 2005; 128:1868-1873.

$\checkmark 9$ Schmid H, Schiffl H: Erythropoiesis-stimulating agents and anaemia of end-stage renal disease. Cardiovasc Hematol Agents Med Chem 2010;8:164-172.
10 Riedel MK, Morgenstern T: Iron replacement in hemodialysis patients with a normal serum ferritin level. Dtsch Med Wochenschr 2004;129:1849-1853.

11 Jacobs C, Frei D, Perkins AC: Results of the European Survey on Anaemia Management 2003 (ESAM 2003): current status of anaemia management in dialysis patients, factors affecting epoetin dosage and changes in anaemia management over the last 5 years. Nephrol Dial Transplant 2005;20:iii3-iii24.

12 KDOQI Clinical Practice Guidelines and Clinical Practice Recommendations for Anemia in Chronic Kidney Disease. Am J Kidney Dis 2006;47:S58. 